\title{
Influence of $\mathrm{Fe}^{3+}$ Ion Substitution on Thermal and Dielectric Properties of Titanium Dioxide
}

\author{
Shirajahammad M. Hunagund, Pradeep Chavan, Vani R. Desai, L.R. Naik, \\ J.S. Kadadevarmath and A.H. Sidarai \\ Department of Studies in Physics, Karnatak University, Dharwad-580003, Karnataka, India \\ *E-mail: ashok_sidarai@rediffmail.com
}

Keywords: AFM, FT-IR, Thermal analysis, Dielectric constant, DSSC.

\begin{abstract}
Thermal and dielectric characteristics of $\mathrm{Fe}_{2} \mathrm{O}_{3}$ doped $\mathrm{TiO}_{2}$ (FDT) nanopowder is investigated. Formation of FDT was prepared using the formula [1-x] $\mathrm{TiO}_{2}+[\mathrm{x}] \mathrm{Fe}_{2} \mathrm{O}_{3}$ by solid state reaction method. The Surface morphology was studied using atomic force microscopy (AFM) technique; it reveals the average particle size in the range of 180 to $200 \mathrm{~nm}$. FT-IR analysis reveals weak transmittance bands between 1020 to $1650 \mathrm{~cm}^{-1}$ and 2800 to $3500 \mathrm{~cm}^{-1}$ which show a gradual decrease in peak intensities with an increase of Fe concentration. Thermal stability was assessed by Thermogravimetric analysis (TGA). Results of TGA profiles indicate that all FDT nanopowder was found to be thermally stable between $400^{\circ} \mathrm{C}$ to $600^{\circ} \mathrm{C}$. The dielectric behavior of the said composite has been investigated as a function of frequency $(20 \mathrm{~Hz}-1 \mathrm{MHz})$ at room temperature and it is observed that the dielectric constant decreases with increasing frequency indicating dispersion behavior.
\end{abstract}

\section{INTRODUCTION}

Thermal properties of the materials have been studied extensively to understand the nature of mechanism involved in products and decompositions. There are several characterization procedures to examine thermal properties, here TGA has been used to studying the path of thermal decomposition. The dielectric relaxation with extremely high permittivity has been reported in many materials, in which the contribution of conduction mechanism (electrons, holes, ions, and protons) to dielectric polarization is one amongst many mechanisms and it plays an important role in the capacitor, ionic batteries, and electrochemical sensor fabrication. Titanium dioxide and ferric oxide were used as a photocatalyst in a wide range of environmental processes due to their unique properties. These semiconductors have the band gap about $3.2 \mathrm{eV}$ and $2.1 \mathrm{eV}$ respectively and possess strong oxidative ability and non-toxicity [1]. Some of the metal ions like $\mathrm{Ag}^{+}$and $\mathrm{Fe}_{3}^{+}$, when used as additives, have provoked an additional antibacterial activity of the titanium photocatalyst [2, 3]. From the last few decades, many researchers studied the dielectric and conduction behavior of ceramic composites and these shows interesting electrical and dielectric properties [4]. Recently these composite materials were used in Dye-sensitized solar cells (DSSC) [5] to enhance the photoelectric conversion efficiency. Here in the thermal properties of FTD were carried out for the decomposition and the dielectric constants as a function of the frequency of FTD were also studied.

\section{EXPERIMENTAL}

The metal oxides with the general formula $[1-\mathrm{x}] \mathrm{TiO}_{2}+[\mathrm{x}] \mathrm{Fe}_{2} \mathrm{O}_{3}$ (in which $\mathrm{x}=0.00,0.05$, $0.10)$ were prepared by solid state reaction method. High purity AR grade materials such as titanium dioxide $\left(\mathrm{TiO}_{2}\right)$, ferric Oxide $\left(\mathrm{Fe}_{2} \mathrm{O}_{3}\right)$ were used to prepare the composites. These metal oxides were taken according to stoichiometric proportion and mixed in liquid medium (acetone medium) to obtain a homogeneous mixture. The composite was grounded in an agate mortar for a longer duration to reduce the particle size. The finely grounded powder is then pre-sintered at $800^{\circ} \mathrm{C}$ for 8 
hours in a programmable muffle furnace and cooled to room temperature. The pre-sintered powder is grounded again and mixed 2-3 drops of PVA (Polyvinyl alcohol) as a binder and pressed into the form of pellets using a hydraulic press. These pellets were finally sintered at $1150^{\circ} \mathrm{C}$ for 12 hours and cooled to room temperature to complete the solid state reaction. Surface morphology and average particle size of the composite was studied by AFM (Model: Nanosurf AG-easy scan2). IR studies were used to get information about the position of the ions in the crystal lattice through the crystal vibrational modes with FTIR spectrophotometer in the range $4000-400 \mathrm{~cm}^{-1}$ (Model: NICOLET-6700). TGA is a technique used to study the thermal stability and decomposition of composites (Model: SDT Q600). The dielectric behavior of the composite has been investigated as a function of frequency in the range $20 \mathrm{~Hz}-1 \mathrm{MHz}$ at room temperature (Model: PSM1700 LCR Meter).

\section{RESULTS AND DISCUSSION}

The particle size of the composite was measured using AFM technique and it was found in the range from 180 to $200 \mathrm{~nm}$. The AFM images for $\mathrm{x}=0.00, \mathrm{x}=0.05$ and $\mathrm{x}=0.10$ are shown in Fig. 1, Fig. 2 and Fig. 3 respectively. The characteristic stretching and bending modes of vibration for the chemical bonds of samples can be effectively evaluated by a spectroscopic method. FT-IR spectra reveal some weak transmittance bands between 1020 to $1650 \mathrm{~cm}^{-1}$ and 2800 to $3500 \mathrm{~cm}^{-1}$ and it also shows a gradual decrease in peak intensities with increasing Fe concentration. These bands are attributed to the stretching vibration of $\mathrm{O}-\mathrm{H}$ groups and bending vibration of absorbed water molecules [6]. Further, the bands Between 500 to $830 \mathrm{~cm}^{-1}$ may be due to different vibrational modes of $\mathrm{TiO}_{2}$, the broad intense band at $1200 \mathrm{~cm}^{-1}$ may be due to Ti-O-Ti vibration as shown in Fig. 4.

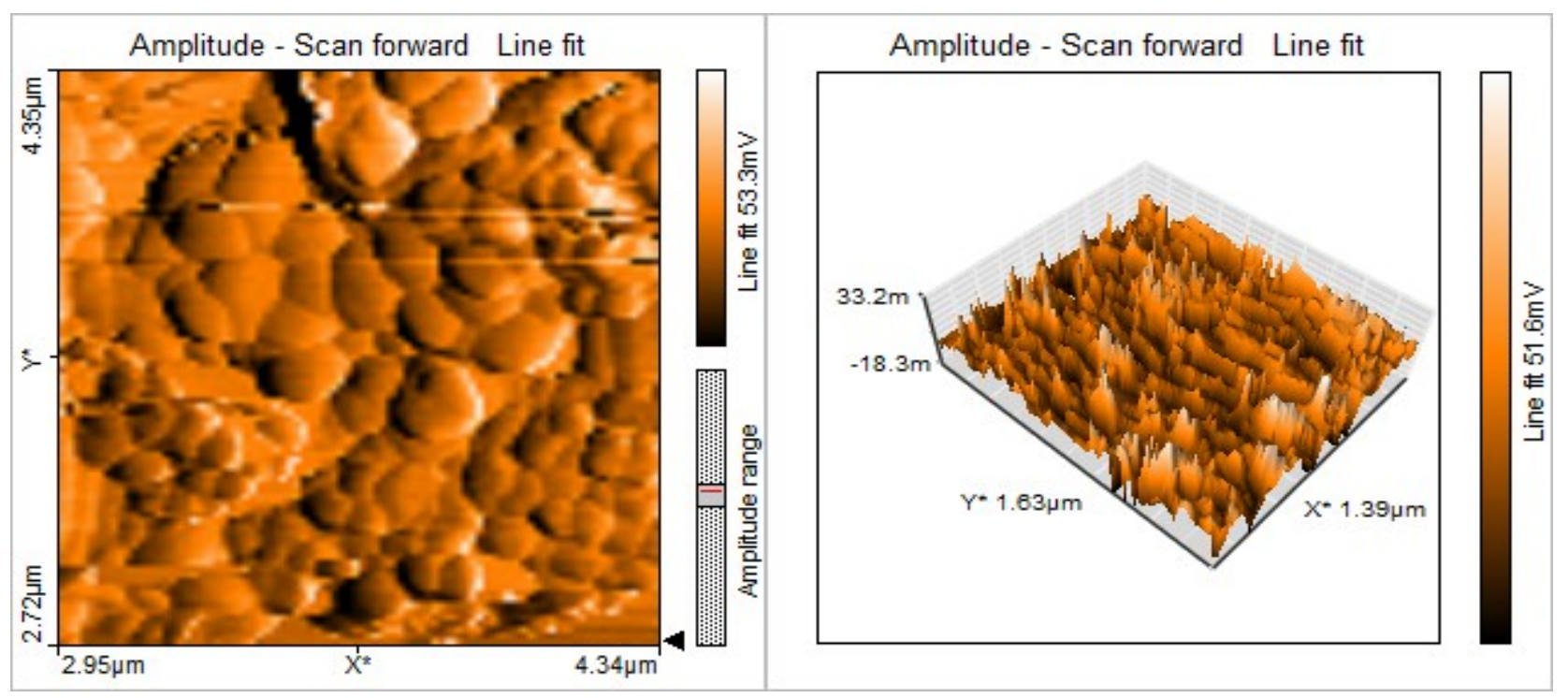

Fig. 1: Colour map and 3D View of AFM image for $x=0.00$. 


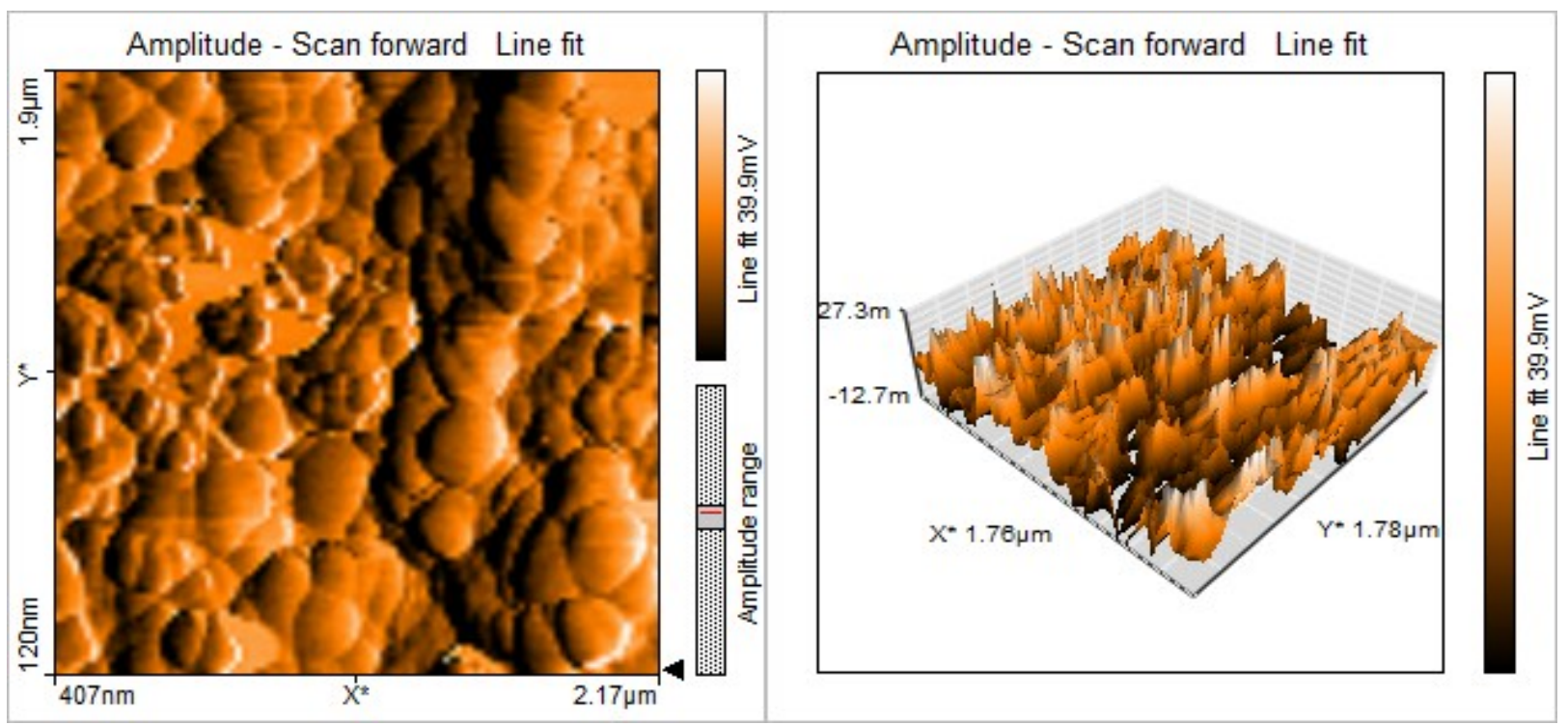

Fig. 2: Colour map and 3D View of AFM image for $x=0.05$.

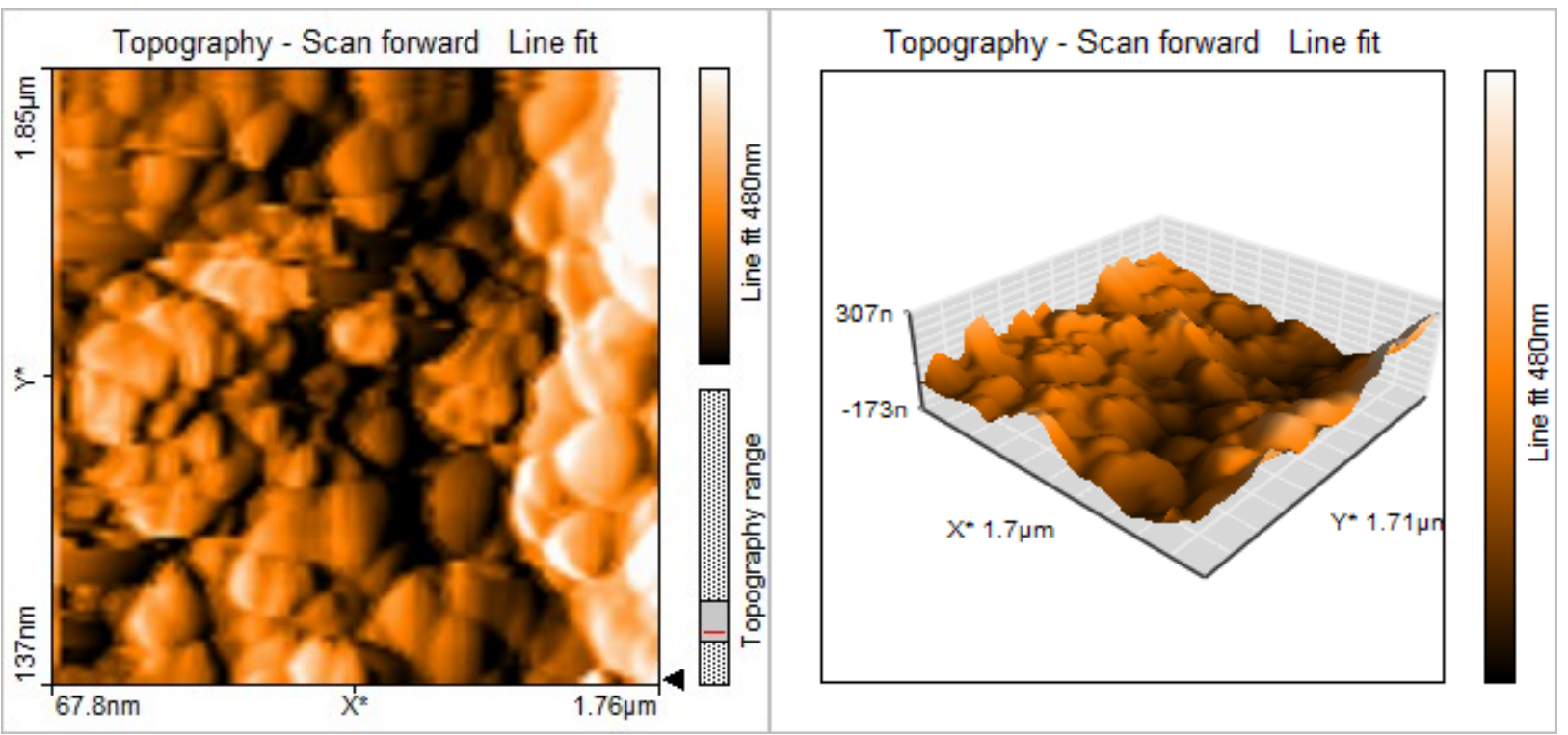

Fig. 3: Colour map and 3D View of AFM image for $x=0.10$.

The thermogram of FDT is presented in Fig. 5. A close look at this figure reveals that all the weight losses occurred may be due to their corresponding thermal events from ambient to $400^{\circ} \mathrm{C}$. After $400^{\circ} \mathrm{C}$, none of the materials underwent greater than $5 \%$ weight loss. Furthermore, with an increase of $\mathrm{Fe}$ content in $\mathrm{TiO}_{2}$, it appears that there was a gradual increase in the weight loss and the corresponding endothermic nature below $400^{\circ} \mathrm{C}$. These results suggest that Fe doping causes the increased absorption of moisture by $\mathrm{TiO}_{2}$ powder. Thus, it may be unequivocally concluded that the FDT powder is thermally quite stable between $400^{\circ} \mathrm{C}$ to $600^{\circ} \mathrm{C}$.

The variation of dielectric constant $(\varepsilon)$ as a function of frequency is shown in Fig. 6. The decrease in dielectric constant with increasing frequency shows dielectric dispersion behavior for all the three composites. The maximum dielectric constant was observed for $\mathrm{x}=0.00$ i.e. pure $\mathrm{TiO}_{2}$ nanopowder shows maximum as compared to other two volume fractions. The dielectric constant was strongly dependent on the composition and here in decreased with the addition of ferric oxide. Therefore, increasing of $\mathrm{Fe}$ ions content causes a decrease in the polarization, which is accomplished by a decrease in dielectric constant, which is attributed to the fact that the electron exchange between $\mathrm{Fe}^{2+}$ and $\mathrm{Fe}^{3+}$ ions cannot follow the change of the external field beyond a certain frequency. 


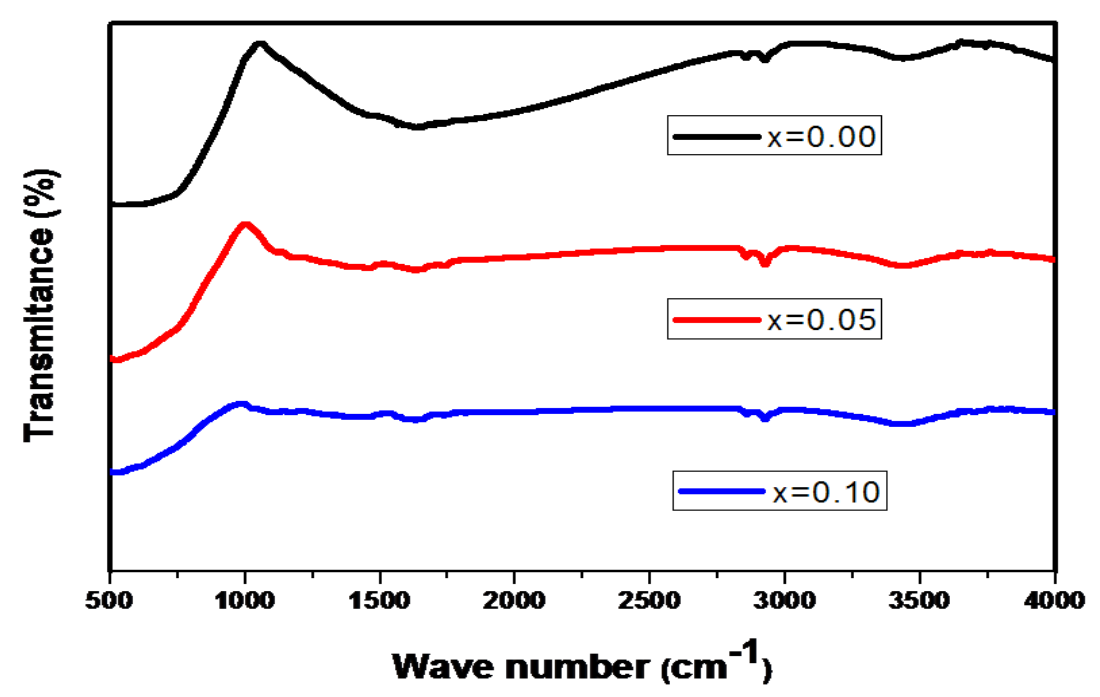

Fig. 4: IR spectra for various composites.

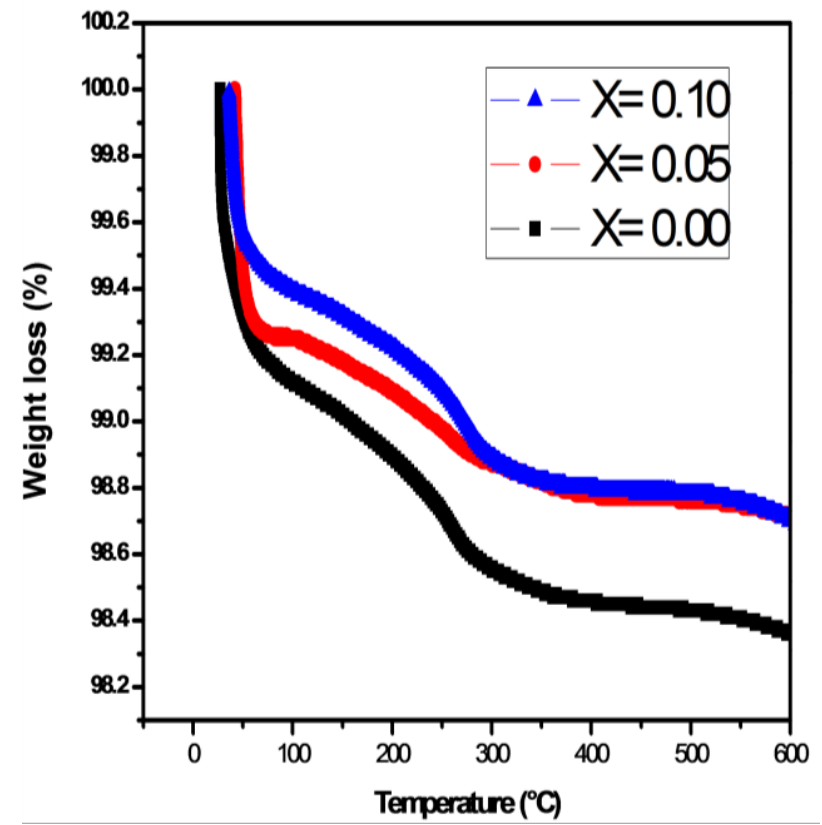

Fig. 5: TGA plots for composites.

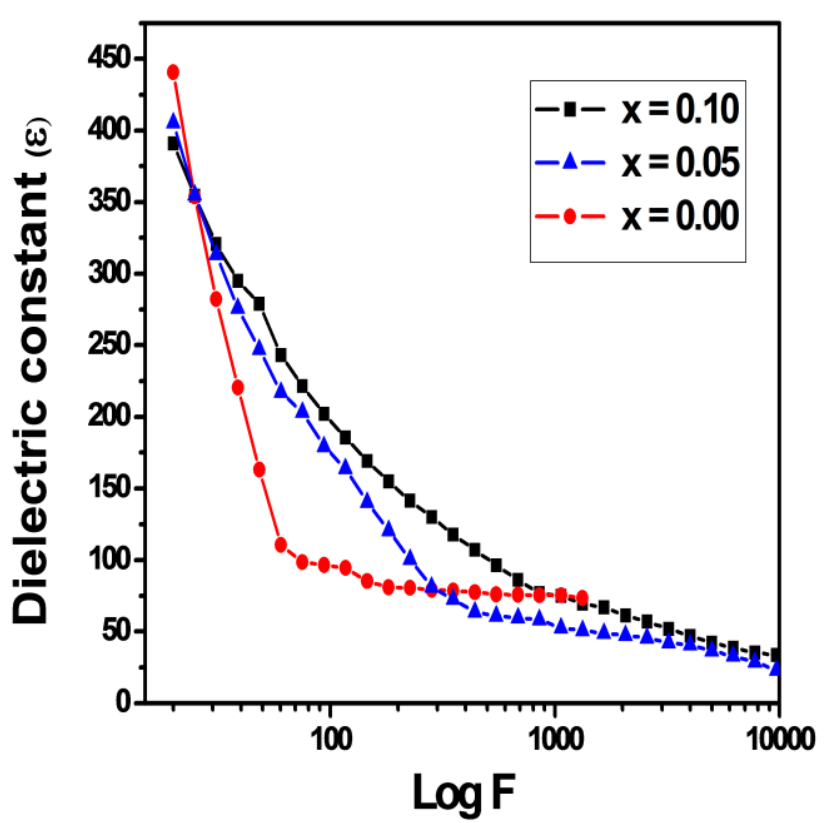

Fig. 6: Variations of dielectric constant as a function of frequency for the composites.

As frequency increases, the dielectric constant attains a constant value and remains independent beyond higher frequencies. These results suggest that composites exhibit dispersion due to Maxwell-Wagner [7,8] type interfacial polarization which may be in agreement with Koop's phenomenological theory [9]. The higher values of dielectric constant observed at lower frequencies may be explained on the basis of space charge polarization as a result of the inhomogeneous dielectric structure. The inhomogeneities in the present composites may be attributed to impurities, porosity and grain structure presence $[10,11]$. But at higher frequencies, the dielectric constant remains independent of frequency may be due to the inability of electric dipoles to follow the fast variation of alternating applied electric field and accordingly the friction between them will increase. A quantity of heat will thus be generated and internal viscosity of system, as well as dielectric constant, may decrease [12]. It is evinced from Fig. 6 that the values of dielectric constant for the constituent phases along with their composites vary in a random fashion at lower frequencies. 


\section{CONCLUSIONS}

From the study, the formation of FDT nanopowder for different volume fractions has been successfully prepared by solid state reaction method. The samples have been characterized by AFM and FTIR. AFM analysis indicates particle sizes were in the range 180 to $200 \mathrm{~nm}$, suggesting nano nature of the composites. FTIR analysis mainly reveals the gradual decrease in peak intensities with increasing Fe concentration. The TGA analysis may unequivocally suggest that FDT composites were thermally stable as compared to pure $\mathrm{TiO}_{2}$. Variation of dielectric constants with respect to frequency shows that the dielectric dispersion behavior occurs at lower frequencies and the dielectric constants were found to decrease with increase in frequency. It is proposed to study the solar energy harvesting property of the said composites.

\section{ACKNOWLEDGEMENTS}

This research work is financially supported by UGC-UPE fellowship from Karnatak University Dharwad. Authors are grateful to the technical staff of the USIC, Karnatak University Dharwad for recording the AFM images, FT-IR spectra, and Thermogravimetry analysis.

\section{REFERENCES}

[1] A.Fujishma, T.N.Rao, D.A.Tryk, Titanium dioxide photocatalysis, J. Photochem. Photobiol. C1 (2000) 1-21.

[2] X.Hou, H.Ma, F.Liu, J.Deng, Y.Ai, X.Zhao, D Mao, D Li, B Liab, Synthesis of Ag ionimplanted $\mathrm{TiO} 2$ thin films for antibacterial application and photocatalytic performance. J. Hazs.Material, 299 (2015) 59-66.

[3] W.Zhang, W.Chen, Yu.S. Chen, Yin.Y, Preparation and antibacterial behavior of $\mathrm{Fe}^{3+}$-doped nanostructured $\mathrm{TiO}_{2}$ thin films. Thin solid films, 516 (2008) 4690-4694.

[4] P.B.Belavi, G.N.Chavan, L.R.Naik, Grain size dependent dielectric and magnetic properties of (y)NCCF+(1-y)bto particulate composites. Int. J. Nanosci. 11 (2012) 1240007 1-5.

[5] Y.Y.Li, H.S.Hao, L.Qin, H.L.Wang, M.Q.Nie, Z.Q.Hu, G.S.Lio. Synthesis and characterization of $\mathrm{Ho}^{3+}$-doped strontium titanate downconversion nanocrystals and its application in dye-sensitized solar cells. J. Alloys. Comp. 622 (2015) 1-7.

[6] I.Ganesh, P.Kumar et al. Preparation and characterization of Fe-doped TiO2 powders for solar light response and photocatalytic applications. Processing and application of ceramics, 6 (2012) 21-36.

[7] J.C.Maxwell, Electricity and Magnetism. Oxford University Press, London (1993) 828.

[8] K.W.Wagner. The theory of incomplete dielectricity. Ann. Phys, 40 (1993) 53.

[9] C.G.Koop.On the dispersion of resistivity and dielectric Constant of Some Semiconductors at Audiofrequencies. Phys. Rev. B, 83 (1951) 121.

[10] D.C. Agarwal. Relaxor ferroelectric materials. Asian J. Phys. 6 (1997) 108.

[11] M.A. El-Hiti. Dielectric behavior and ac electrical conductivity of Zn-substituted Ni, Mg ferrites. J. Magn. Magn. Mater. 164 (1996) 187-196.

[12] M.A.Ahmed, S.T.Bisay, G.Abdelatif. Effect of ytterbium on the electrical properties of $\mathrm{Li} \pm \mathrm{Co}$ ferrite. J. Phys. Chem. Solids, 62 (2001) 1039-1046. 